\title{
Questioni di limite: predicare la misura
}

Questions de limite : prêcher la mesure

Limit Issues: Preaching the Measure

\section{Maria Giuseppina Muzzarelli}

\section{OpenEdition}

\section{Journals}

Edizione digitale

URL: http://journals.openedition.org/cei/5832

DOI: $10.4000 /$ cei.5832

ISSN: 2260-779X

\section{Editore}

UGA Éditions/Université Grenoble Alpes

\section{Edizione cartacea}

ISBN: 978-2-37747-159-1

ISSN: 1770-9571

\section{Notizia bibliografica digitale}

Maria Giuseppina Muzzarelli, «Questioni di limite: predicare la misura», Cahiers d'études italiennes

[Online], 29 | 2019, online dal 30 septembre 2019, consultato il 27 mars 2021. URL: http:// journals.openedition.org/cei/5832 ; DOl: https://doi.org/10.4000/cei.5832

Questo documento è stato generato automaticamente il 27 mars 2021

(c) ELLUG 


\title{
Questioni di limite: predicare la misura
}

\author{
Questions de limite : prêcher la mesure \\ Limit Issues: Preaching the Measure
}

Maria Giuseppina Muzzarelli

1 È vero che l'efficacia si misura dagli effetti, effetti della predicazione nel caso specifico ${ }^{1}$, ma la si può ragionevolmente ipotizzare anche esaminando le intenzioni, gli argomenti addotti dal predicatore, il suo modo di ragionare sull'uno o sull'altro tema, il rapporto del predicatore con il suo pubblico e con le autorità cittadine.

2 Il tema che vorrei qui affrontare, per ragionare sull'efficacia delle parole di alcuni predicatori, è quello dell'invito alla misura, alla moderazione, la raccomandazione cioè a non superare limiti peraltro non ben specificati. Tema trattato in città nelle quali le leggi suntuarie ${ }^{2}$ fissavano precisi limiti alle esibizioni e dunque anche ai consumi.

3 La relazione fra l'azione dei legislatori e le parole dei predicatori è l'elemento efficace o almeno da me supposto come tale e corrisponde all'idea che punire senza educare è vano. Così, mentre i legislatori vietavano o dosavano (capi di abbigliamento, accessori, portate ai banchetti e così via), i predicatori inducevano a ragionare su limiti e misure, educavano alla moderazione. «Doimmè, misuratevi, misuratevi un poco!» esortava $\mathrm{i}$ Senesi nel 1427 Bernardino da Siena ${ }^{3}$. L'opera sia dei legislatori sia dei predicatori era verosimilmente funzionale alla governabilità, esprimeva l'intenzione di facilitare la convivenza in contesti compositi e conflittuali nei quali la smisuratezza più che la disuguaglianza rischiava di aggiungere problemi a problemi.

4 Si trattava dunque di indurre i cittadini a rispettare ordine e gerarchia anche nell'abbigliarsi, cosa che dalla metà del XIII secolo presero a fare gli Statuti con specifici provvedimenti suntuari. Si trattava di indurli a stare al proprio posto e a non eccedere rispetto a una misura che le leggi indicavano con una certa precisione e alla quale $\mathrm{i}$ predicatori si limitavano ad alludere. Questi ultimi invitavano a non superare i limiti rivolgendosi a una società urbana che tutto o quasi contava e misurava e che faceva 
corrispondere posizioni elevate a grandi misure e l'armonia urbana al rispetto delle proporzioni e delle gerarchie e quindi delle differenze.

5 L'idea è quella di collegare le parole dei predicatori in fatto di limite e misura allo stesso scopo della governabilità perseguita dalle leggi suntuarie. Cosa serve per governare una città nella quale, a partire da almeno il XIII secolo, la partecipazione al potere era un dato di fatto e la lotta per la partecipazione una realtà drammatica $\mathrm{e}$ quotidiana ${ }^{4}$ ? Cosa serve a facilitare la convivenza in ambiti nei quali le differenze anche enormi erano all'ordine del giorno ed in contesti caratterizzati dalla ineguaglianza asserita e sottolineata all'interno di un preciso ordinamento gerarchico?

6 Ai governanti servivano molte doti ed altrettanti strumenti, servivano certamente efficaci mezzi di convincimento oltre a incisivi mezzi di coercizione. Serviva anche un'opera continua e sottile di costruzione del consenso. Serviva lavorare sull'idea che la differenza è un dato naturale, la gerarchia un fatto inevitabile e che la misura è necessaria per affrontare le diversità e sopportare le differenze gerarchiche. Teorizzare e sostenere l'importanza della misura comportava condannare ogni effetto estremo, tanto la troppa povertà come l'eccessiva ricchezza, sia lo sciupio vistoso sia l'accumulo avaro. Molti degli elementi dei discorsi che seguiranno ritorneranno su questi argomenti e su questi termini.

7 Il tema della misura è centrale nelle leggi suntuarie intese come strumento di governo, come mezzo per fissare e rendere riconoscibili le differenze, come occasione per ribadire la gerarchia: fra le diverse posizioni sociali, fra i generi, persino fra le parti del corpo. Si pensa a queste leggi come mezzo per limitare il lusso e già questo ha a che fare con l'idea del contenimento e della misura, ma queste leggi si sono occupate anche di manifestazioni estetiche che non hanno o quasi rapporto con il lusso e che erano invece importanti per riconoscere le diverse posizioni sociali nonché per mantenere e rendere visibili le gerarchie. Per queste ultime due finalità assume capitale importanza il tema della misura o meglio della commisurazione, della proporzione cioè fra quello che si è e quello che si esibisce, fra distanze sociali e differenze anche estetiche.

8 Nei testi delle norme suntuarie le misure ricorrono in continuazione: misure esatte, numeriche accanto a generici richiami alla moderazione. I legislatori fissavano il numero dei capi che le appartenenti alle diverse categorie sociali potevano possedere, il peso dei bottoni, l'altezza del bordi di pelliccia, la lunghezza dello strascico: una vera e propria orgia di numeri e di limiti. Nella normativa bolognese del $1401^{5}$, per limitarci a questo unico esempio, sono concesse al massimo, senza operare distinzioni o quasi fra le categorie sociali, tre once d'argento o d'oro filato come ornamento della testa, dodici once e non più nelle vesti e nelle decorazioni, sei once in cordelle, quindici once nella cintura. L'altezza della pancia del vaio, vale a dire circa 6 o 7 centimetri, era un limite invalicabile per le bordature in pelliccia, il numero massimo di anelli consentiti era $3, \mathrm{i}$ profili delle sopravvesti non potevano essere più alti di un'oncia (circa 1/10 di piede $\mathrm{e}$ cioè $3 \mathrm{~cm}$ ), le maniche non più larghe di due braccia (cioè circa $1,30 \mathrm{~m}$, braccio circa $65 \mathrm{~cm}$ ) «mensurando circumcirca» e non più lunghe di un braccio, compresa la mano della donna che indossava la veste. Ma le maniche delle mogli e delle figlie di cavalieri o dottori potevano raggiungere la larghezza di due braccia e mezzo (dunque mezzo braccio in più rispetto alle altre donne e quindi circa 30 centimetri segnalavano il privilegio). I bordi in pelliccia delle maniche non potevano superare «tres dossi varorum in longitudine» e i manicotti sotto alle maniche non potevano essere più lunghi del braccio della donna e più larghi di tre once. Nessuna veste poteva avere una 
circonferenza, all'altezza dei piedi, di oltre dieci braccia 6-7 metri. Quanto alla lunghezza non poteva superare quella della donna con le pianelle. La punta delle pianelle non poteva essere più lunga di mezza oncia. In alcuni casi il limite è dato dal valore dell'oggetto: non si poteva portare nessun «paternoster» che valesse più di 15 lire, pena la perdita dell'oggetto. Chi possedeva capi «contra legem» li poteva continuare a portare (ma non per Quaresima) se provvedeva immediatamente a denunciarli e bollarli. Dunque i legislatori avevano le idee abbastanza chiare in fatto di limiti e misure.

Il ragionamento dei predicatori sulla necessaria misura («non oltre») solo in qualche caso faceva riferimento al disciplinamento suntuario ma sappiamo che la connessione esisteva ed era ricercata; tuttavia i predicatori sostenevano la necessità e il valore della moderazione a prescindere dal disciplinamento suntuario attuato per legge e a partire da una conoscenza piuttosto esatta degli atteggiamenti e dei gusti degli uomini e delle donne dei loro tempi nonché degli oggetti che il mercato rendeva disponibile esercitando una forte attrazione non solo sulle donne, come invece i predicatori tendevano a far credere.

10 Il trattato di Giovanni da Capestrano Degli ornamenti specie delle donne, base per prediche sul tema ${ }^{6}$, risulta essere stato composto in soccorso delle politiche suntuarie coeve in particolare contro gli strascichi in occasione della lotta ingaggiata al riguardo a Ferrara negli anni Trenta del XV secolo per contenere il culto smodato delle apparenze. Un collegio di giuristi e di predicatori richiese appunto l'intervento di Giovanni da Capestrano. Il suo ragionamento prende avvio dal pensiero di san Tommaso secondo il quale l'ornamento non è peccato in sé ma per l'uso che se ne fa, tanto che il vizio sopravviene nell'usarne immoderatamente ${ }^{7}$. La posizione del capestranese appare più severa: l'uso dell'ornamento esteriore è ordinariamente proibito e comunque più che misurato ha da essere commisurato, proporzionato cioè alla dignità e all'ufficio ${ }^{8}$. Sono tre i criteri per ritenere ragionevole un ornamento: la dignità della persona, la consuetudine del paese, la nobiltà dell'ufficio e ogni volta che, ornandosi, si sorpassa la necessità e la convenienza si cade nel vizio della superfluità̀. Quest'ultima è rappresentata da dimensioni eccessive, ad esempio da pianelle di altezza genericamente indicata come immoderata ${ }^{10}$. Incrociando fonti di tipo diverso siamo in grado di chiarire quando l'altezza delle pianelle era immoderata: lo era, stando alle indicazioni contenute nei provvedimenti suntuari emanati in diverse città d'Italia, quando il rialzo superava le $4-5$ dita cioè $7-8 \mathrm{~cm}$. A Venezia i legislatori hanno rappresentato graficamente la pianella per loro ideale con indicazione esatta del sopralzo consentito che non doveva superare appunto le $5 \mathrm{dita}^{11}$. Questa era l'altezza generalmente permessa: così a Perugia come a Terni ma anche a Firenze o a Todi. A noi può sembrare poca cosa ma all'epoca l'altezza del tacco a piattaforma doveva costituire questione di non trascurabile rilievo (forse anche in considerazione della quantità di tessuto in più che comportava un alto sopralzo con relativa esibizione di ricchezza ma anche di inutile sciupio) se, come accadde a Orvieto, determinò reazioni cittadine che portarono a concessioni. Grazie a queste ultime, dal limite di 4 dita si arrivò, con una revisione statutaria, a una totale liberalizzazione: «fuit reformatum quod unicuique liceat portare planellas ad sui libitum quo ad altitudinem $»^{12}$.

11 Il capestranese dunque criticava l'altezza immoderata delle pianelle senza precisare misure ma indicando come moderata e dunque giusta quella funzionale, utile cioè a riparare dal fango e dalla polvere. Se maggiore, configurava vizio e delineava una forma 
di contraffazione menzognera. Bernardino da Feltre ha fatto cenno a un caso del genere riferendo di un marito che credeva di avere scelto una donna bene in carne che invece, eliminate le imbottiture, risultò magra («Et tu si Deus fecit te macram, te voi far grassa cum straze e peze et maritus, qui credebat accipere un pezo de carne, ha tolto strazo e peze» $)^{13}$. Anche a un marito che credeva di avere una moglie alta poteva capitare di scoprirla di bassa statura una volta fatta scendere da pianelle con elevata piattaforma. Nel caso si sarebbe delineato, seguendo Bernardino da Feltre, un imbroglio analogo a quello realizzato da un artigiano che spacciasse una merce per un'altra («artifex qui vendit unum pro altero»).

12 A chiarire le idee sull'altezza delle pianelle intervennero, come si è detto, i legislatori: oltre le 4-5 dita erano smisurate a prescindere dalla condizione sociale di appartenenza. Diverso il discorso sugli strascichi, la lunghezza dei quali era spesso proporzionata alla posizione sociale e variavano da 1 braccio a $2 / 3$ di braccio, $1 / 2$ braccio e $1 / 3$ di braccio. Per le calzature «eccessive», interveniva ad aggravare la colpa, agli occhi dei predicatori, l'applicazione dell'ornamento a parti abbiette, i piedi ${ }^{14}$, il che fa riferimento alla opportunità di tenere conto, nell'ornarsi, della gerarchia fra le parti del corpo. Nel caso invece degli strascichi, l'aggravante era rappresentata dalle numerose conseguenze negative, anche igieniche, del trascinamento in terra di costoso e prezioso tessuto. Bernardino da Siena ne ha parlato diffusamente in una predica latina, "Contra se fardantes et capillos adulterinos portantes, atque contra feminas caudatas» ${ }^{15}$.

13 A voler estrapolare dalle osservazioni del Capestranese elementi di una teoria generale dell'ornamento consentito, direi che per essere ragionevole doveva «star lontano» da superfluità, curiosità e inonestà, ma anche lontano dal «troppo» e seguire «la via del mezzo». La stessa teoria si applicava anche agli apparati della casa tanto che il letto, ad esempio, non doveva essere troppo molle, troppo prezioso o troppo grande ${ }^{16}$. Così il Capestranese ha operato per diffondere e radicare una mentalità della moderazione, per sviluppare una coscienza critica rispetto all'eccesso, al troppo, allo spreco, all'inutile e all'accumulo.

Di accumulo vizioso o meglio infruttuoso ed anzi dannoso parla Bernardino da Siena, ragionando su una coppia di concetti e termini in opposizione fra loro: «ragunare» versus «spargere». La predica XLI del ciclo del 1427 è l'occasione per riflettere sul «troppo», sul possedere e ammassare più del necessario sviluppando un pensiero sulla ricchezza che configura una particolare sintesi fra economia e crematistica ${ }^{17}$.

Bernardino da Siena non condanna la ricerca del profitto in sé e dunque la moltiplicazione delle risorse se si tratta o si ha in mente di perseguire il bene della collettività. Bernardino ben conosce l'interesse e la passione per il guadagno che anima il suo pubblico e tende a condurre questo atteggiamento nella direzione del benessere della comunità che poteva essere aumentato da abili mercanti, avveduti agricoltori o attenti investitori se capaci di ridistribuire. Sulla base di questo assunto anche la «moltiplicazione della roba» va bene e può far bene quando prevede l'azione dello «spargere». In questo caso perfino l'eccesso può essere virtuoso, la illimitatezza augurabile, l'atteggiamento avido accettabile. Ed ecco che Bernardino legittima lucrosi guadagni e buoni affari se finalizzati a dispensare ricchezza anziché ad accumulare risorse:

E però ti dico: se vuoi che la tua robba multiplichi, usa di dare limosine. Sai come fa questa limosina, o tu che raguni el letame, sai, fuor della porta? Pon mente che mai 
in su questo letame non vi nasciarà grano. Sai perché? Perché egli è amontato: se tu lo spargesse, non sarebbe così. Va' e spargelo sopra a uno terreno che sia magro, e seminavi su, e vedrai quanto frutto elli ti rendarà ${ }^{18}$.

16 Fuor di metafora, Bernardino in più prediche (ad es. XLI e XLII) critica il «ragunare la robba», quando fine a se stesso. In una pagina dell'edizione delle prediche tenute sul Campo de' Fiori nel 1427, il termine «ragunare» ricorre quattro volte ${ }^{19}$, tre volte nella pagina successiva e due e più volte per pagina in quelle che vengono dopo. L'oggetto polemico è l'accumulo dell'avaro che mai si contenta, mai si sazia: è l'esempio concreto della dismisura che fa male alla comunità ma anche a lui stesso. Diverso l'atteggiamento nei confronti della ricchezza distribuita.

17 Ne aveva parlato nella predica XXXVIII sui mercanti e sul fare mercanzie, attività lecita se fatta con l'animo giusto e avendone bisogno (di nuovo il tema della misura: quando e quanto se ne ha bisogno?): «Sappi che per niuno modo non t'è lecito aragunare, se non per colui che n'ha bisogno» ${ }^{20}$.

18 C'è poi chi accumula non per avidità ma mosso dalla preoccupazione del futuro, per quando, dice un ipotetico interlocutore, "gli verrà a bisogno». Ma quanto credi di vivere? gli risponde il predicatore. «Questa non è altro che cupidità» e sottrazione ai poveri di quanto loro spetta ${ }^{21}$. Bernardino ricorda in più occasioni l'obbligo a dare ai poveri tutto quello che supera il bisogno anche se non propriamente superfluo. Va detto che ciò che supera il bisogno è indicazione davvero generica. Meno generico il riferimento a quanti vanno aiutati distinguendoli in tre categorie: i bisognosi, i necessitosi e coloro che si trovano «in istremità». A questi ultimi si è obbligati a prestare aiuto perché essi, diversamente da quanti hanno abbondanza oppure sufficienza di beni, hanno assoluta necessità e

[...] tu se' tenuto da quello che ti bisogna in su, di dare a chi n'ha bisogno e a chi n'ha necessità e a chi è in estremità [...] fa' che tu non dica «Io mi voglio serbare quello che è di bisogno per me: s'io invecchiasse, io non potrei guadagnare, io n'ho bisogno io per me. $\mathrm{O}$ s'io vivesse dugento o trecento anni?» $[. . .]^{22}$.

Sempre in dialogo con l'ipotetico interlocutore, quest'ultimo gli fa presente che Oh, s'io desse de la mia robba a chi mi viene a le mani, io ispergiarei ogni cosa in pochissimo tempo, e verrei in quella miseria d'avere poi bisogno io! [...] io dico che tu dia di quello che è dal tuo bisogno in su a chi n'ha bisogno; [....] a chi ha bisogno, a chi ha necessità e a chi è in stremità [... ${ }^{23}$.

19 Bernardino rende suggestivo il suo discorso sulla mal distribuzione dei beni facendo riferimento a cassoni talmente colmi di grano da non poterlo «governare» e a donne che hanno pieni i "goffani» di panni o a persone che hanno tanti denari da non sapersene cosa fare:

O tu che hai in casa tanto grano ragunato, pieni e granai, pieni e palchi, pieni e cassoni, e per la tanta quantità non potendolo governare, elli si viene a guastare, e elli sel mangiano e pontaruoli, e anco le passare n'hanno la lor parte, e 'l povaro n'ha bisogno $[. . .]^{24}$.

20 L'assunto di Bernardino è che chi ha «robba in abondanzia» la deve dare «a chi n'ha necessità e bisogno» ${ }^{25}$, anche perché l'accumulo non è senza conseguenze, come dimostra il caso di un ortolano che aveva costume di «dare per Dio ciò che esso avanzava da la sua vita in su». Quando, invecchiando, cambiò atteggiamento e "cominciò a ragunare per sé», finì che tutto quel denaro che prima distribuiva ai bisognosi lo dovette spendere in medici e in medicine ${ }^{26}$. Nel corso del racconto delle pene dell'ortolano, che arrivò anche a subire l'amputazione di un piede, la parola 
«ragunare» compare quattro volte: è il fulcro del discorso, il centro dell'episodio, il cuore della questione.

21 Se «ragunare» è un male che fa male (una cancrena come quella che ha colpito l'ortolano), «spargere» è un bene che fa bene, dà ricchi frutti, è come prestare a usura a Iddio che renderà «cento per uno»: la limosina «multiplic[a] la robba», se l'abbondante letame è «ragunato» non nasce il grano, ma se tu lo spargi su un terreno anche magro, «vedrai quanto frutto ti rendarà $»^{27}$.

Misurare, limitare, distribuire sono tre raccomandazioni che tornano anche nel discorso di Bernardino sulle vanità, dove si fa esplicito riferimento anche alle leggi suntuarie (con fatica i Consigli cercano di porre rimedio a che la gente non si metta tanto oro addosso, tanto panno per vestire, non faccia maniche grandi come ali per volare all'inferno $\left.{ }^{28}\right)$. Dice Bernardino: «[...] egli t'è lecito di serbarti il tuo bisogno, ma dal bisogno tuo in là tu debbi sovenire il povaro per amore di Dio» ${ }^{29}$. Se Dio ti ha dato tanta abbondanza, è perché tu ne sia dispensatore («spargere»): non puoi possedere senza peccato il «superchio» ${ }^{30}$.

Il tema è di nuovo quello dell'accumulo versus la distribuzione, dove accumulo significa eccesso, dismisura, uso avaro delle cose: un insieme di vizi pericolosi per la vita cittadina. Si cade in simili colpe anche amando le vanità che offendono Dio in dieci modi «per cagione de vestimenti»: quando ci si veste oltre la «misura» del proprio status, quando si eccede in varietà o in «suavità» e via elencando. Offende Dio «chi si veste di quello che non apartiene a lui» e, aggiunge Bernardino, «[...] dico che il ricco die vestire onorato più che l'artefice, sì bene: ma non voler vestire tanto onorato, che tu passi il termine» ${ }^{31}$.

24 Tanto l'argomento dell'iniquità come quello della superfluità, due delle dieci offese a Dio comportate dalle vanità, hanno rapporto con la misura, anzi con la dismisura e quindi con la sproporzione di chi, ad esempio, «accumula» vesti che deve difendere dalle «tignuole» mentre la poveretta muore di freddo: «Elli si conviene che sia d'altrui, quello che si possede di superchio [...] dal bisogno tuo in là tu debbi sovenire il povaro [...] debbi dare con discrezione» ${ }^{32}$.

Dio, esorta il predicatore, ti ha dato tanta abbondanza di beni «perchè tu sia suo dispensatore $»^{33}$. L'accumulo, infruttuoso per definizione per Bernardino da Siena, arreca danno alla città: «Meglio ti sarebbe che quelli danari tu gli mettesse ne la tua bottiga in mercantia [...] $»^{34}$.

Segue un discorso sulla dannosità comportata dagli eccessi (in definitiva sinonimo di vanità) applicati alle diverse parti del corpo: per i piedi il ragionamento si incentra sullo spreco economico comportato da alte pianelle. I sopralzi eccessivi, osserva il predicatore, producono ingenti lievitazioni dei costi: se un paio di pianelle in sé costerebbe da mezzo fiorino a un fiorino, può finire con il costarne 6,18 e perfino 60 se si valuta il prezzo del tessuto in più che l'altezza delle pianelle comporta:

El più largo del vestire è ne le pianelle, però che quanto più se' presso a terra, più è largo il vestimento: una spanna di quello da pie' ne porta più di quatro di quello da capo. E quello che ne va ne la coda, ch'io avevo lassato ${ }^{35}$ ?

Anche i predicatori non scherzano in fatto di numeri e misure!

Aveva esordito il Senese nel suo discorso sui vestimenti corporali dicendo «Vuoi tu vedere quanto è mala cosa?». Ecco che mezzo secolo dopo, Bernardino da Feltre sembra idealmente ripartire da lì, dal fatto che «la brigata non se ne fa stima» del tema delle pompe e delle vanità che invece «non sono cossì de pocha stima como pensii» ${ }^{36}$. Del 
resto Bernardino da Siena aveva parlato di «disfacimento d'una città la vanità de le donne, per li velluti e drappi, chè se tali denari stessero in su le mercanzie, vi farebbero assai utilità, e voi le tenete ne' goffani ${ }^{37}$.

Anche fra gli argomenti addotti da Bernardino da Feltre vi è la critica ai denari lasciati morti nelle vanità mentre i poveri muoiono di fame: ciò configura l'omicidio ${ }^{38}$ mentre in un'altra predica ragiona sulla liceità di vestire in maniera commisurata al proprio status:

Ben non ne licet mihi induere me secundum statum meum? Sum doctor, nobilis, civis de bona casa. E cossì dico io: Sta secundum statum tuum. - Quid importat stare? - Id est dritto. Sta rectus non curvus, non gibbosus. Ez. 2,1: Sta super pedes tuos. Sta fermo, saldo, non camminar. Quid est ergo status tuus? Stare rectus [...] considerando tempus preteritum, presena et futurum ${ }^{39}$.

La commisurazione dell'abito al proprio status implica la considerazione della provenienza per nascita e delle funzioni in svolgimento:

0 , dicit iulle, sum dux, comes, eques, doctor, civis nobilis etc., habeo divitias et. Dei gratia, el posso fare. - Attende. Maior in honore nobilioribus, dignioribus et preciosioribus uti deent vestimentis [...]. Si sunt in officio ministeriatus, veste de Signori durante officio, sed expleto officio, ad sua exercitia cum vestibus moderatis [...]. Chi è la persona più degna del mondo ${ }^{40}$ ?

Chi vuole andare oltre il proprio status crede di andare in su e invece va in giù, precipita all'inferno: «Va' zo, va' zo [...] Serva ergo ordinem, [...] Quia non servasti ordinem, descende in profundum inferni, ibi nullus est ordo» ${ }^{41}$.

Commisurare e «tenere il mezzo» sono le regole indicate. Nella graduatoria delle manchevolezze l'eccedere è il male più grave, peccato mortale, mentre «non facere tantum quantum potes» è peccato veniale ${ }^{42}$. L'eccesso è il «superchio»: «Superchio in valore, superchio in politeza, superchio si una vestis sufficit, doe, tre, quattro superfluunt» ${ }^{43}$.

L'indicazione della moderazione è largamente presente sia nel pensiero laico sia in quello religioso, compare nei proemi delle leggi suntuarie ma anche nei ragionamenti di filosofi come di mercanti avveduti, ciò da Aristotele a Beniamino Franklin, ma anche prima e dopo tali rilevanti, seppure diversissimi, protagonisti del pensiero.

31 Quello che caratterizza l'azione dei predicatori di cui ci siamo qui occupati è l'impegno a trasformare il principio della moderazione in cultura corrente e diffusa, anche a sostegno dei legislatori. Questi ultimi parlavano, ad esempio nei proemi delle leggi bolognesi, di intollerabili e immoderate spese, di spese sontuose e inordinate, di fatuità e stoltizia delle donne e di molti uomini che hanno moglie, di necessità di ovviare alle vesti e agli ornamenti immoderati e sontuosi delle donne ${ }^{44}$. A tali dichiarazioni generali facevano seguito misure precise da rispettare pena una multa, una punizione fisica, il sequestro del bene e perfino l'imprigionamento.

Predicare la moderazione significava sviluppare la coscienza della sostenibilità, come si usa dire oggi, proporre e valorizzare un modo di atteggiarsi discreto, misurato funzionale anche all'accettazione del disciplinamento civico che per essere accolto aveva bisogno dell'intervento efficace dei predicatori. I contributi dei predicatori miravano alla creazione di una cultura del rispetto delle gerarchie e quindi anche del privilegio di pochi, ma nella disponibilità da parte di questi pochi a distribuire «dal bisogno [...] in là». La ridistribuzione era anche uno degli scopi dei legislatori che cercarono di attuarla attraverso le multe. 
Misura predicata dagli uomini di Chiesa e misure imposte dai legislatori si sono fiancheggiate e talvolta hanno collaborato a fini di efficacia per garantire governabilità e cioè equilibrio, se non armonia, nella differenza gerarchica. L'azione congiunta ha assicurato l'efficacia, impedendo alle parole dei predicatori di essere pure esortazioni ed ha fatto dell'indicazione alla misura uno strumento politico ma anche un mezzo per radicare la coscienza dei consumi, per far crescere il ragionamento sull'uso della ricchezza che le norme cercavano di condizionare almeno un po', se non di dirigere. Alcuni corpi di leggi si dichiarano esplicitamente frutto dell'azione di predicatori evidentemente efficaci. Così le Riformanze di Orvieto del 1468 che attribuiscono a Francesco da Viterbo l'idea di prendere provvedimenti restrittivi in consiglio; così a Terni nel 1507, dove le determinazioni prese in materia di doti e di vesti muliebri sono ricondotte alle esortazioni di un frate dell'Osservanza che evidentemente aveva predicato con frutto, e così nelle Riformanze tudertine del 1575 dove si legge che un predicatore, evidentemente efficace, aveva indirizzato all'uditorio, autorità cittadine comprese, «spaventevoli minacce» che indussero a provvedere contro disordini e superfluità consiglio e vivido descrittore delle vanità femminili e delle esibizioni di oro, argento e perle: seguirono provvedimenti restrittivi ${ }^{45}$. Non ci sono noti i nomi di molti di questi efficaci predicatori che probabilmente non erano fra i più famosi all'epoca ma sicuramente erano preparati nell'indagare gli eccessi ed efficaci sostenitori delle misure imposte per legge quando non autonomamente osservata dagli uomini e soprattutto dalle donne.

Predicare la misura e limitare le esibizioni, e dunque i consumi, sono le due facce di quella ricerca di armonia che appare alla base delle regole rinascimentali per le costruzioni di palazzi e piazze: ben proporzionate, misurate appunto come quella de «La Città ideale» dipinta tra il 1470 e il 1490. Dietro alla proposta di un ideale del genere ma anche dietro ad effettive realizzazioni, Pienza per esempio, ci sono secoli di ragionamenti su misura e moderazione ed esperimenti di limiti da imporre e da introiettare. L'armonioso e quasi magico equilibrio comunicato dal famoso dipinto di autore anonimo è più sognato che reale, del resto durava poco anche l'effetto delle leggi suntuarie che cercavano di disciplinare desideri e consumi e verosimilmente durava ancora meno l'impressione suscitata dalle parole dei predicatori.

Lo squilibrio, la sproporzione, il troppo di pochi e il poco di troppi (in definitiva l'ingiustizia) rappresentano forse il più grande dei problemi sociali dell'oggi e forse non è del tutto inutile ragionare, a proposito di limiti e di misure, su cosa può rendere efficace ogni azione di contrasto: l'opera congiunta dell'educatore e del legislatore, normare e insieme insegnare formando una durevole coscienza dei limiti a partire dai propri.

\section{BIBLIOGRAFIA}

BERNARDINO DA SIENA, Quadragesimale de christiana religione, in Opera omnia, t. II, Firenze, Quaracchi, 1950. 
BERNARDINO DA SIENA, Le prediche volgari. Predicazione del 1425 in Siena, a cura di C. Cannarozzi, 2 voll., Firenze, Rinaldi, 1958.

BERnARDino DA SIENA, Prediche volgari sul Campo di Siena, 1427, a cura di C. Delcorno, 2 voll., Milano, Rusconi, 1989.

Giovanni Da CAPESTRAno, Degli ornamenti specie delle donne, a cura di A. Chiappini, Siena, Cantagalli, 1956.

KovesY Catherine, Sumptuary Law in Italy, 1200-1500, Oxford, Clarendon Press, 2002.

HunT Alan, Governance of the Consuming Passion. A History of Sumptuary Law, New York, Palgrave Macmillan, 1996.

MUZZARELli Maria Giuseppina, Gli inganni delle apparenze. Disciplina di vesti ed ornamenti alla fine del medioevo, Torino, Scriptorium-Paravia, 1996.

MuZZARELli Maria Giuseppina (a cura di), La legislazione suntuaria. Secoli XIII-XVI. Emilia-Romagna, Roma, Ministero per i Beni e le Attività Culturali, Direzione generale per gli Archivi, 2002.

Muzzarelli Maria Giuseppina, Le leggi suntuarie, in C. M. Belfanti e F. Giusberti (a cura di), Storia d'Italia. La moda, Annali 19, Torino, Einaudi, 2003, pp. 180-220.

MuzZARELl Maria Giuseppina, «De ornatu mulierum». Il caso delle pianelle, in G. P. Brizzi e G. Olmi (a cura di), Dai cantieri della storia. Liber amicorum per Paolo Prodi, Bologna, CLUEB, 2007, pp. 435-444.

MuZzARELli Maria Giuseppina (a cura di), From Words to Deeds. The Effectiveness of Preaching in the Late Middle Ages, Turnhout, Brepols, 2014.

Nico OtTAVIAni Maria Grazia (a cura di), La legislazione suntuaria. Secoli XIII-XVI. Umbria, Roma, Ministero per i Beni e le attività culturali. Direzione generale per gli Archivi, 2005.

VARISCHI DA MILANo Carlo (a cura di), Sermoni del b. Bernardino Tomitano da Feltre, 3 voll., Milano, Cassa di Risparmio delle Provincie lombarde, Banca del Monte, 1964.

\section{NOTE}

1. M. G. Muzzarelli (a cura di), From Words to Deeds. The Effectiveness of Preaching in the Late Middle Ages, Turnhout, Brepols, 2014.

2. Ead., Le leggi suntuarie, in C. M. Belfanti e F. Giusberti (a cura di), Storia d'Italia. La moda, Annali 19, Torino, Einaudi, 2003, pp. 180-220.

3. Bernardino da Siena, Prediche volgari sul Campo di Siena, 1427, a cura di C. Delcorno, Milano, Rusconi, 1989, predica XXXVII, pp. 1068-1098, in partic. p. 1075.

4. A. Hunt, Governance of the Consuming Passion. A History of Sumptuary Law, New York, Palgrave Macmillan, 1996. Vedere anche: M. G. Muzzarelli, Gli inganni delle apparenze. Disciplina di vesti ed ornamenti alla fine del medioevo, Torino, Scriptorium-Paravia, 1996; C. Kovesy, Sumptuary Law in Italy, 1200-1500, Oxford, Clarendon Press, 2002.

5. M. G. Muzzarelli (a cura di), La legislazione suntuaria. Secoli XIII-XVI. Emilia-Romagna, Roma, Ministero per i Beni e le Attività Culturali, Direzione generale per gli Archivi, 2002, Bologna, 1401, gennaio, Statuto suntuario, pp. 127-136.

6. Giovanni da Capestrano, Degli ornamenti specie delle donne, a cura di A.Chiappini, Siena, Cantagalli, 1956. Lo spunto per scrivere questo trattato gli venne probabilmente dall'incarico che Giovanni ricevette nel 1434 dal vescovo di Ferrara, Giovanni da Tossignano, di trattare il tema 
degli strascichi che preoccupavano sia le autorità religiose sia quelle civili: M. G. Muzzarelli, Gli inganni dell apparenze, cit., pp. 175-182.

7. Giovanni da Capestrano, op. cit., p. 20.

8. Ivi, p. 75.

9. Ivi, pp. 131 e 88.

10. Ivi, pp. 100 e 117.

11. M. G. Muzzarelli, «De ornatu mulierum». Il caso delle pianelle, in G. P. Brizzi e G. Olmi (a cura di), Dai cantieri della storia. Liber amicorum per Paolo Prodi, Bologna, CLUEB, 2007, pp. 435-444.

12. M. G. Nico Ottaviani (a cura di), La legislazione suntuaria. Secoli XIII-XVI. Umbria, Roma, Ministero per i Beni e le attività culturali. Direzione generale per gli Archivi, 2005, Orvieto, Statuti 1537, p. 1075.

13. C. Varischi da Milano (a cura di), Sermoni del b. Bernardino Tomitano da Feltre, 3 voll., Milano, Cassa di Risparmio delle Provincie lombarde, Banca del Monte, 1964, vol.1, sermone 38, pp. 475-487, in partic. p. 477.

14. Giovanni da Capestrano, op. cit., pp. 97-98.

15. Bernardino da Siena, Quadragesimale de christiana religione, in Opera omnia, Firenze, Quaracchi, 1950, t. II, sermo XLVII, pp. 86-99.

16. Giovanni da Capestrano, op. cit., p. 127.

17. Bernardino da Siena, Prediche volgari sul Campo di Siena, 1427, cit., predica XLI, pp. 1202-1230.

18. Ivi, p. 1221.

19. Ivi, in partic. p. 1235.

20. Ivi, predica XXXVIII, pp. 1099-1138, in partic. p. 1110 ma vedere anche p. 1108.

21. Ibid.

22. Ivi, predica XL, pp. $1169-1201$, in partic. p. 1187.

23. Ibid.

24. Ivi, pp. 1188-1189.

25. Ivi, p. 1187.

26. Ivi, p. 1216.

27. Ivi, rispettivamente pp. 1219 e 1221.

28. Ivi, predica XXXVII, p. 1087.

29. Ivi, p. 1083.

30. Ivi, p. 1085: «serba quello che bisogna a te e a' tuo' figliuoli, e l'avanzo da' al bisognoso»; ogni «superchio» dispiace a Dio.

31. Ivi, p. 1076.

32. Ivi, p. 1083.

33. Ibid.

34. Ivi, p. 1088.

35. Ivi, p. 1095.

36. Sermoni del b. Bernardino Tomitano da Feltre, cit., sermone 38, p. 475.

37. Bernardino da Siena, Le prediche volgari. Predicazione del 1425 in Siena, a cura di C. Cannarozzi, 2 voll., Firenze, Rinaldi, 1958, vol. II, predica XXVIII, pp. 82-97, in partic. p. 95.

38. Sermoni del b. Bernardino Tomitano da Feltre, cit., vol. I, sermone 38, p. 484

39. Ivi, vol. II, sermone 100, «De vanitatibus et pompis», pp. 121-131, cit. p. 122.

40. Ivi, p. 123.

41. Ivi, p. 124

42. Ivi, p. 125.

43. Ibid.

44. Le leggi suntuarie. Secoli XIII-XVI. Emilia-Romagna, cit. Bologna: vedere Proemi alle disposizioni suntuarie bolognesi del 1335 (p. 78), del 1376 (p. 106), del 1389 (p. 116) o del 1398 (p. 122) ma anche del 1401 (p. 127) e del 1453 (p. 148). 
45. La legislazione suntuaria. Secoli XIII-XVI. Umbria, cit.; vedere rispettivamente alle pp. 1028 (Orvieto, 1468, maggio 5, Riformanze), 839 (Terni, 1507, maggio 30, Riformanze), 805 (Todi, 1575, marzo 20, Riformanze), 1040 (Orvieto, 1473, aprile 21, Riformanze).

\section{RIASSUNTI}

In più casi i predicatori dell'ultimo Medioevo hanno alluso ad una necessaria misura nei consumi che possono e devono corrispondere alle necessità, scontatamente diverse per le ineguaglianze esistenti, mantenute e giustificate, di persone di differente «status» e condizione economica. Consumi diversi, sì, ma non oltre misura, appunto, non oltre il bisogno, anzi i bisogni.

Si vuole ragionare brevemente sul concetto di limite («non oltre») in alcuni predicatori o trattatisti la cui riflessione e produzione di pensiero si connette al disciplinamento suntuario. Si vuole riflettere su cosa indicano questi pensatori in fatto di misura e se si può ricavare, da quanto da loro indicato, qualche elemento rivelatore della loro idea di governabilità nella pur necessaria differenza e nella dovuta distinzione.

À plusieurs reprises, certains prédicateurs de la fin du Moyen Âge ont réclamé une nécessaire mesure dans la consommation, qui peut et doit correspondre au besoin, sans pour autant négliger les inégalités existant entre les individus, dont le «status » et la condition économique varient. Cette consommation doit certes être différenciée, mais elle ne doit jamais dépasser la juste mesure et la nécessité.

Cet article vise à étudier le concept de limitation («non oltre ») chez certains prédicateurs ou auteurs de traités, dont la réflexion et la pensée se rattachent à la discipline somptuaire. Son objectif est d'observer ce qu'indiquent ces penseurs en matière de mesure et de tirer de leurs indications des éléments révélateurs de leur doctrine en la matière.

On several occasions, some preachers of the late Middle Ages have called for a necessary measure in consumption, which can and must correspond to the need, without neglecting the inequalities between individuals, whose "status" and economic condition are different. This consumption must of course be differentiated, but it must never go beyond just measure and necessity.

This article aims to study the concept of limitation in certain preachers or authors of treatises, whose reflection and thought are related to the sumptuary discipline. Its purpose is to observe what these thinkers indicate in terms of measurement and to draw indications revealing their doctrines in the matter.

\section{INDICE}

Mots-clés : prédication, Bernardin de Sienne, Bernardin de Feltre, lois somptuaires, mesure, limite, mode vestimentaire

Keywords : preaching, Bernardino of Siena, Bernardino of Feltre, sumptuary laws, measure, boundary, fashion dress

Parole chiave : predicazione, Bernardino da Siena, Bernardino da Feltre, leggi suntuarie, misura, limite, moda vestimentaria 
AUTORE

MARIA GIUSEPPINA MUZZARELLI

Université de Bologne.

maria.muzzarelli@unibo.it 